\title{
Transient damage spreading and anomalous scaling in mortar crack surfaces
}

\author{
Stéphane Morel, ${ }^{1, *}$ Daniel Bonamy, ${ }^{2}$ Laurent Ponson, ${ }^{3}$ and Elisabeth Bouchaud ${ }^{2}$ \\ ${ }^{1}$ US2B, UMR 5103, Université Bordeaux 1, 351 Cours de la Libération, 33405 Talence Cedex, France \\ ${ }^{2}$ DSM/DRECAM/SPCSI, CEA-Saclay, 91130 Gif-Sur-Yvette Cedex, France \\ ${ }^{3}$ Division of Engineering and Applied Science, California Institute of Technology, Pasadena, California 91125, USA
}

(Received 19 July 2007; revised manuscript received 13 May 2008; published 23 July 2008)

\begin{abstract}
The scaling properties of a post-mortem mortar crack surface are investigated. The root mean square of the height fluctuations is found to obey anomalous scaling properties, but with three exponents, two of them characterizing the local roughness $\left(\zeta \simeq 0.79\right.$ and $\left.\zeta_{e} \simeq 0.41\right)$ and the third one driving the global roughness $\left(\zeta_{g} \simeq 1.60\right)$. The critical exponent $\zeta \simeq 0.79$ is conjectured to reflect damage screening occurring for length scales smaller than the process zone size, while the exponent $\zeta_{e} \simeq 0.41$ characterizes roughness at larger length scales, i.e., at length scales where the material can be considered as linear elastic. Finally, we argue that the global roughness exponent could be material dependent contrary to both local roughness exponents $(\zeta \simeq 0.8$ and $\zeta_{e} \simeq 0.4$ ) which can be considered as universal.
\end{abstract}

DOI: 10.1103/PhysRevE.78.016112

PACS number(s): 62.20.M-

\section{INTRODUCTION}

Since the pioneering work of Mandelbrot et al. [1], the statistical characterization of fracture surfaces has been a very active field of research. The fracture surfaces of various materials have shown surprising scaling properties (see Bouchaud [2,3] for reviews) in the sense that they exhibit self-affine scaling invariance over a wide range of length scales. A large amount of studies focused on the statistical characterization of fracture surfaces obtained in materials as different as metallic alloys [4-7], ceramics [8,9], glass $[10,11]$, quasicrystals $[12,13]$, rocks $[14,15]$, mortar $[16,17]$, sea ice [18], and wood $[19,20]$ have shown self-affine scaling properties characterized by a local roughness exponent $\zeta$ $\approx 0.8$ and this in spite of huge differences in the fracture mechanisms. It was therefore suggested that this local roughness exponent $\zeta$ might have a universal value [4], i.e., independent of the fracture mode and of the material.

However, quite recently, significantly different values of the local roughness exponent $\zeta$ have been measured. First, different values of the local roughness exponent have been found due to the anisotropy and the heterogeneity of the material structure [7,21-23], the kinetics of crack growth [24] or the possible multifractal character of the crack surfaces [25]. Second, fracture surfaces were shown to exhibit anisotropic scaling morphological features, characterized by two different roughness exponents whether observed along the direction of crack front or the direction of crack growth $[10,12,13]$. Beyond this simple scaling analysis, the whole two-dimensional (2D) structure function-when properly nondimensionalized - was shown $[12,13]$ to take a universal specific form irrespective of the considered material, the failure mode, and the crack growth velocity. Third, recent experiments in sandstone [26,27], artificial rock [28], and granular packing of sintered glass beads with various bead sizes and porosities investigated on a wide range of crack growth velocities $[11,29]$ have shown self-affine scaling

\footnotetext{
*s.morel@us2b.u-bordeaux1.fr
}

properties, especially at large length scales, characterized by a roughness exponent measured along the direction of crack front significantly smaller than 0.8 , closer to $0.4-0.5$. This suggests the existence of a second universality class for failure problems.

A possible interpretation of these two universality classes was proposed recently [11]. It was indeed reported that 0.8 roughness exponent was measured in materials where surfaces were observed at length scales below the size of the process zone $(\mathrm{PZ})$ while the 0.4 roughness exponent was estimated at length scales above the PZ size. Such an interpretation is funded on recent results obtained from two distinctive models. Bonamy et al. derived a model from linear elastic fracture mechanics (LEFM) that predicts 0.4 selfaffine fracture surfaces for perfectly brittle materials, i.e., in the absence of any damage and/or plastic deformations [11]. Thus, the 0.4 roughness exponent could reflect the roughness of fracture surfaces at length scales where the material can be approximated as a linear elastic medium and as a consequence at length scales above the PZ size. On the other hand, using a paradigm of the mode I fracture model (quasistatic fuse model), Hansen and Schmittbuhl [30] have recently suggested that the universality of 0.8 roughness exponent could be due to the fracture propagation being a damage coalescence process described by a stress-weighted percolation phenomenon in a self-generated quadratic damage gradient. In this sense, the 0.8 roughness exponent could reflect the roughness of fracture surfaces at length scales below the PZ size, i.e., where such a damage percolation process can take place. However, the two regimes had never been observed on the same fracture surface. It is the central point of this letter to show that both regimes are observed on a mortar fracture surface.

We focus on the transient roughening regime developing from a straight notch which has been suggested [31] to be associated to the process zone development and to the socalled $R$-curve effect. We will show that both local roughness exponents can be observed on the same profile, the two regimes coexisting in the presence of anomalous roughening. 


\section{EXPERIMENT}

The studied fracture surface is obtained from a mortar notched beam subjected to four points bending, leading to a mode I failure $[16,17]$. The length of the beam is $1400 \mathrm{~mm}$ and its height and thickness are both equal to $140 \mathrm{~mm}$. The initial notch is performed with a steel sheet (thickness 0.4 $\mathrm{mm}$ ) pulled out when the mortar is $24 \mathrm{~h}$ old. The notch length is fixed to $70 \mathrm{~mm}$, which corresponds to one-half of the beam height. The specimen geometry leads to a mode I stable crack growth for the first $10-15 \mathrm{~mm}$ of the crack. Within this range of crack lengths, fracture is quasistatic. On the other hand, the mortar is constituted by a sand for which the grain size ranges between $0.1 \mathrm{~mm}$ and $1.5 \mathrm{~mm}$, and by a high strength Portland cement.

The topographies of the fracture surfaces are recorded using an optical profilometer. In all of the following, the reference frame $(\vec{x}, \vec{y}, \vec{z})$ is chosen so that $\vec{x}, \vec{y}$, and $\vec{z}$ are parallel to the propagation, loading and crack front directions, respectively [the average plane of the crack surface corresponds to the $(\vec{x}, \vec{z})$ plane]. The maps are built up with 300 profiles of 4096 points parallel to the initial notch ( $z$ direction). The sampling step $\Delta z$ along profiles is $20 \mu \mathrm{m}$. The first profile $(x=0)$ is sampled in the immediate vicinity of the initial straight notch tip and so corresponds to a quasizero roughness. The distance between two successive profiles $\Delta x$ is fixed to $50 \mu \mathrm{m}$. As the distance $x$ to the initial notch increases, the magnitude of the roughness develops up to 7 $\mathrm{mm}$. The lateral precision (along the $x$ and $z$ directions) is $2 \mu \mathrm{m}$, while the vertical accuracy ( $y$ axis), estimated from the height differences between two successive profiles along the same line, is approximately $5 \mu \mathrm{m}$.

\section{A. Local roughness regimes}

The roughening of the crack profiles can be estimated through the root mean square (rms) $\Delta h(l, x)$ of the height $\left\{h\left(z_{i}, x\right)\right\}_{1<i<N_{l}}$ inside a window of size $l$ along the $z$ axis and averaged over all possible origins $j$ of the window belonging to the profile

$$
\Delta h(l, x)=\left\langle\frac{1}{N_{l}} \sum_{i=1}^{N_{l}} h\left(z_{i}, x\right)^{2}-\left(\frac{1}{N_{l}} \sum_{i=1}^{N_{l}} h\left(z_{i}, x\right)\right)^{2}\right\rangle_{j}^{1 / 2} .
$$

Figure 1 shows a log-log plot of the variations of the rms roughness $\Delta h$ with the window size $l$ for two profiles; the first profile corresponding to $x=x_{1}$ is close to the initial notch while the second one $\left(x=x_{2}\right)$ is far from the notch. On each profile, two distinct behaviors corresponding to two power laws can be observed. The first power law observed at small length scales is characterized by a roughness exponent $\zeta$ $=0.79$, while the second power law, observed at large length scales is characterized by a different roughness index $\zeta_{e}$ $=0.41$. The crossover length scale $\xi$ is defined as the abscissa of the intersection between the fits of both power laws. It is found to increase with the distance $x$ from the initial notch, $\xi\left(x_{1}\right)<\xi\left(x_{2}\right)$ in Fig. 1. In other words, the increasing of the distance $x$ from the initial notch favors the small scales regime related to the roughness index 0.8 , to the detriment of the large scales 0.4 regime.

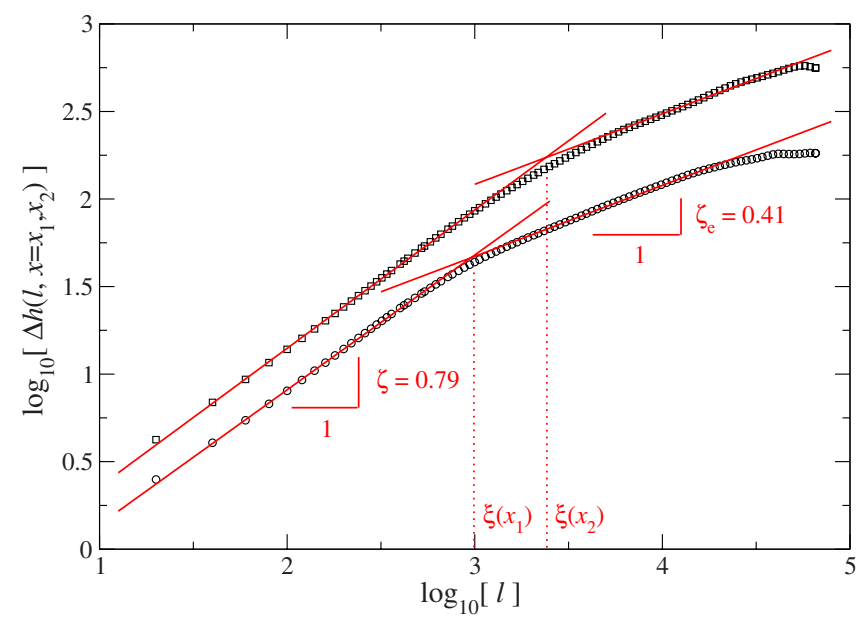

FIG. 1. (Color online) Root-mean-square roughness $\Delta h(l)$ vs $l$ for two profiles located at the beginning $\left(x=x_{1}\right.$, circles $)$ and at the end ( $x=x_{2}$, squares) of the domain of growing roughness. The slopes of both straight lines give estimates of the local roughness exponents $\zeta=0.79$ (for $l \ll \xi$ ) and $\zeta_{e}=0.41$ (for $l \gg \xi$ ). All length scales are given in $\mu \mathrm{m}$.

\section{B. Anomalous roughening}

As can be seen in Fig. 1, the magnitude of the rms roughness of the profile at position $x=x_{2}$ is larger than the one of the profile at position $x_{1}$ for any length scale $l$. This global vertical shift of the $\Delta h(l, x)$ curves as a function of distance $x$ from the initial notch is well known to be symptomatic of an anomalous scaling [16,17,20,21,32,33]. Nevertheless, in the present case, the original anomalous scaling proposed in literature $[20,21,32,33]$ needs to be modified in order to take into account the existence of two local regimes characterized by roughness indices $\zeta \simeq 0.8$ and $\zeta_{e} \simeq 0.4$ (Fig. 1). Let us assume that the crossover length scale $\xi$ between these two regimes scales with the distance $x$ from the initial notch, as a power law characterized by the dynamic exponent $z_{x}$ : $\xi(x)$ $\sim x^{1 / z_{x}}$. On this basis, the original anomalous scaling must be modified in the following way:

$$
\Delta h(l, x) \sim \begin{cases}l^{\zeta} x^{\left(\zeta_{g}-\zeta\right) / z_{x}} & \text { if } l \ll x^{1 / z_{x}} \\ l^{\zeta} x^{\left(\zeta_{g}-\zeta_{e}\right) / z_{x}} & \text { if } l \gg x^{1 / z_{x}}\end{cases}
$$

According to (2), for a given profile, i.e., for a given position $x$, the rms roughness estimated for length scales $l \ll \xi(x)$ $\sim x^{1 / z_{x}}$ is expected to scale as $\Delta h[l \ll \xi(x), x=c t e] \sim l^{\zeta}$ while, for length scales $l \gg \xi(x)$, the roughness scales as $\Delta h[l$ $\gg \xi(x), x=c t e] \sim l^{\zeta_{e}}$, which is in agreement with the results shown in Fig. 1. Moreover, if the roughening is observed as a function of $x$, the roughness estimated at small length scales, i.e., for $l \ll \xi(x)$, is expected to scale as $\Delta h[l$ $\ll \xi(x), x] \sim x^{\left(\zeta_{g}-\zeta\right) / z_{x}}$, while the roughness at large length scales behaves as $\Delta h[l \gg \xi(x), x] \sim x^{\left(\zeta_{g}-\zeta_{e}\right) / z_{x}} . \zeta_{g}$ is referred to as the global roughness exponent, and is different from and independent of both local roughness exponents $\zeta$ and $\zeta_{e}$.

In order to observe the different roughening at small and large length scales, the rms roughness is plotted, in Fig. 2, as a function of the distance $x$ from the initial notch. Note that in Fig. 2, only some window sizes ranging from $l=20 \mu \mathrm{m}$ to 


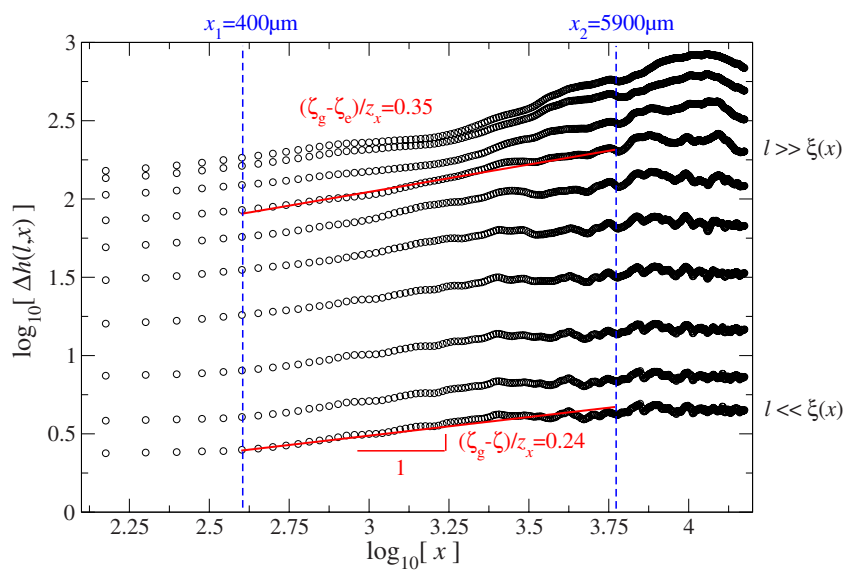

FIG. 2. (Color online) Log-log plot of the rms roughness as a function of the distance $x$ to the initial notch. The part between the positions $x_{1}$ and $x_{2}$ corresponds to the roughness growth domain. According to Eq. (2), the lower straight line corresponds to the fit of the roughness growth observed at small length scales [i.e., for $l$ $\ll \xi(x)]$ and its slope is equal to 0.24 corresponding to the scaling exponent $\left(\zeta_{g}-\zeta\right) / z_{x}$. The upper straight line is related to the roughness growth at large length scales [i.e., for $l \gg \xi(x)]$ and leads to the estimate of the scaling exponent $\left(\zeta_{g}-\zeta_{e}\right) / z_{x}=0.35$.

$60 \mathrm{~mm}$ are kept, for the sake of clarity. The rms roughness $\Delta h$ grows as a function of $x$ from approximately zero for profiles close to the initial straight notch. Note that in Fig. 2, the roughness growth is essentially observed for $x$ values ranging between $x_{1}$ and $x_{2}$ (which correspond to the positions of the profiles plotted in Fig. 1). For distances $x<x_{1}$ $\simeq 400 \mu \mathrm{m}$, the roughness magnitude is approximately constant because the nonzero thickness of the initial notch $(0.4$ $\mathrm{mm}$ ) imposes a nonzero roughness at the onset of crack propagation. For $x>x_{2} \simeq 5.9 \mathrm{~mm}$, the roughness magnitude saturates for a wide range of length scales $l$ (for the largest length scales $l$, the roughness exhibits fluctuations which are doubtlessly due to a slight macroscopic warping of the fracture surface). Within the growing zone, i.e., for distances $x_{1} \leq x \leq x_{2}$, it can be observed that the roughness growth is different if considered for length scales smaller or greater than the crossover length scales $\xi(x)$. In an equivalent way, such different growths can be observed in Fig. 1 from the vertical shifts of the rms roughness observed for position $x_{1}$ and $x_{2}$ which are different if observed at scales $l \ll \xi$ and $l$ $\gg \xi$.

On the other hand, in order to estimate the global roughness index $\zeta_{g}$ and the dynamic exponent $z_{x}$, it is useful to introduce a scaling function describing the behavior of $\Delta h(l, x)$. From the modified anomalous scaling (2), let us define the scaling function $g_{m}(u)$ as $g_{m}\left(l / x^{1 / z_{x}}\right)=\Delta h(l, x) / l^{\zeta_{g}}$. Thus, the modified scaling function $g_{m}(u)$ is expected to scale as

$$
g_{m}(u) \sim \begin{cases}u^{-\left(\zeta_{g}-\zeta\right)} & \text { if } u \ll 1, \\ u^{-\left(\zeta_{g}-\zeta_{e}\right)} & \text { if } u \gg 1,\end{cases}
$$

where $u=l / x^{1 / z_{x}}$. For all profiles corresponding to positions ranging from $x_{1}$ to $x_{2}$ (a total of 110 profiles), the experimental values of $g_{m}(u)$ have been computed for various values of

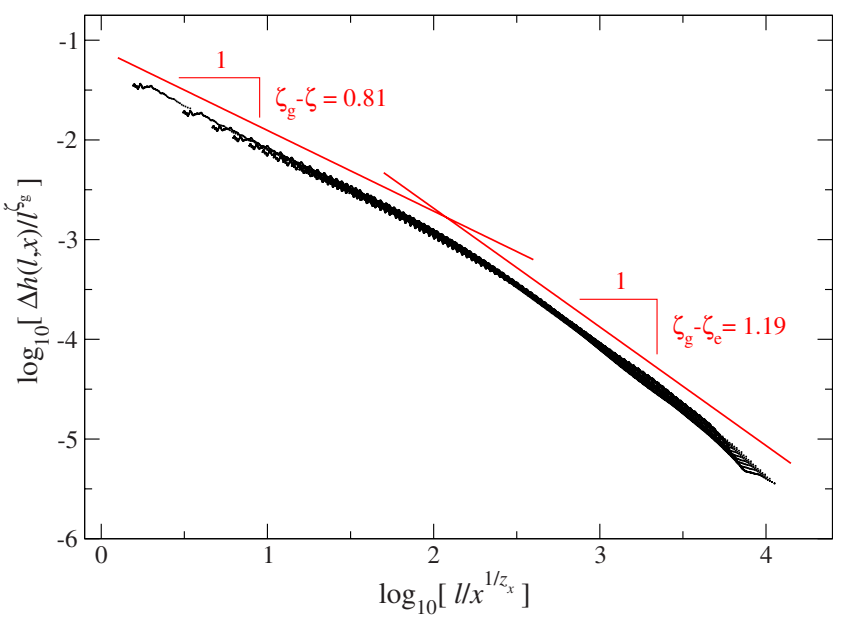

FIG. 3. (Color online) Modified anomalous scaling function $g_{m}(u)$ [Eq. (3)]. The data collapse (corresponding to 110 profiles corresponding to positions $x_{1} \simeq 400 \mu \mathrm{m}$ to $x_{2} \simeq 5.9 \mathrm{~mm}$ ) leads to the exponent values $\zeta=0.79, \zeta_{e}=0.41, \zeta_{g}=1.60 \pm 0.10$, and $z_{x}$ $=3.4 \pm 0.2$.

$\zeta_{g}$ and $z_{x}$, while the values of the local roughness exponents were kept equal to the previous estimates $\left(\zeta=0.79\right.$ and $\zeta_{e}$ $=0.41)$. As shown in Fig. 3, the best data collapse is obtained for $\zeta_{g}=1.60 \pm 0.10$ and $z_{x}=3.4 \pm 0.2$. Note that these values are in agreement with the corresponding values which can be estimated from the slopes of the straight lines plotted in Fig. 2 , the slope of the lower line $(0.24)$ corresponding to $\left(\zeta_{g}\right.$ $-\zeta) / z_{x}$ while the one of the upper line $(0.35)$ is related to $\left(\zeta_{g}-\zeta_{e}\right) / z_{x}$

\section{DISCUSSION}

From the roughness analysis of a mortar crack surface, we have shown that both roughness regimes characterized by the local exponents $\zeta \simeq 0.8$ and $\zeta_{e} \simeq 0.4$ can coexist on the same fracture surface, along the same crack profile. This has led us to modify the original anomalous scaling [32], and the remarkable collapse of the 110 profiles located in the growing zone of roughness (Fig. 3) is consistent with the modified law (2). The two local roughness exponents $\zeta \simeq 0.8$ and $\zeta_{e}$ $\simeq 0.4$ are observed at small and large length scales, respectively. These observations are in agreement with the picture proposed in [11] which suggests that the 0.4 exponent should be observed at length scales - larger than the PZ size-where the material can be approximated as a linear elastic medium, while the 0.8 exponent reflects the presence of damage at length scales smaller than the size of the PZ. In this scenario, the crossover length scale $\xi$ is set by the PZ size or more exactly by a correlation length of the PZ in the direction perpendicular to the crack propagation one. This correlation length could be linked to a correlated gradient percolation process in the PZ as suggested by Hansen and Schmittbuhl [30]. The increase of $\xi$ within the transient roughening regime with the distance $x$ from the initial straight notch is then fully consistent with the quasibrittle fracture behavior of mortar, characterized by a transient initial regime reflecting the increase in size of the microcracked PZ and/or the in- 
crease in microcracks density in PZ before reaching its critical and steady value. In this sense, the extension of the transient roughening regime given by the position $x_{2}$ could reflect the trace of the PZ development (attached to the initial notch tip) before the propagation of the main crack with its critical PZ. Thus, the maximum crossover length scale $\xi_{\max }$ $=\xi\left(x_{2}\right)$ as well as the extension $x_{2}$ of the transient roughening regime could provide post mortem estimates of length scales linked to the critical PZ in the directions perpendicular and parallel to the crack propagation direction. Note that the values of the length scales expected to be linked to the PZ, i.e., $\xi_{\max }=\xi\left(x_{2}\right) \simeq 2.3 \mathrm{~mm}$ and $x_{2}=5.9 \mathrm{~mm}$, are of the same order of magnitude as the estimates of the equivalent LEFM length of PZ given by the crack length increment for which the plateau value of the $R$ curve occurs in mortar [34]. Note also that $\xi_{\max } \simeq 2.3 \mathrm{~mm}$ is slightly larger than the largest grains of sand $(1.8 \mathrm{~mm})$. On the other hand, the dynamic exponent $z_{x}$ could characterize the evolution in size of the microcracked $\mathrm{PZ}$ and/or the increase in microcracks density in $\mathrm{PZ}$ with respect to $x$ through the evolution of the correlation length $\xi(x)$, i.e., $\xi(x) \sim x^{1 / z_{x}}$. In the same way, estimated at the crossover length scale $l=\xi$ according to Eq. (2), the roughness $\Delta h(l=\xi, x) \sim \xi^{\zeta_{g}}\left[\right.$ or $\left.\Delta h(l=\xi, x) \sim x^{\zeta_{g} / z_{x}}\right]$ provides an estimate of the maximum height fluctuations in the PZ, and, indirectly, of the magnitude of the toughness fluctuations and/or microcracks density inside this zone. Thus, the global roughness exponent $\zeta_{g}$ could be the signature of the transient damage spreading and this is why it could be material dependent, contrary to both local roughness indices $(\zeta$ and $\zeta_{e}$ ), which should be considered as universal.

\section{CONCLUSION}

To conclude, one can now reinterpret the values of the roughness exponents-measured for fracture profiles parallel to the direction of crack front-reported in the literature [4,5,7-21,26-29], for various materials.

(i) When the PZ size remains small with respect to the specimen size, as for brittle failure, the crossover length scale $\xi$ is expected to be very small. Then, models derived from linear elastic fracture mechanics such as $[11,35]$ should be able to capture the morphology of fracture surfaces. In particular, such models can reproduce 0.4 roughness regimes in agreement with observations reported in sandstone $[26,27]$, artificial rocks [28], and granular packing of sintered glass beads [29]. In these materials, the 0.8 roughness regime should be difficult to observe, because it is only effective for the smallest length scales. Moreover, damage spreading within the process zone should be scarce, and one expects to observe a classical Family-Vicsek roughening [36] rather than an anomalous scaling [32,33].

(ii) When the process zone size becomes important as compared to the specimen size, as for quasibrittle failure, one expects to observe a large crossover length scale $\xi$. In this case, the 0.8 roughness regime should take place over a wide range of length scales while the 0.4 roughness regime should be only observed for the largest length scales where it can be perturbed by a finite size effect making its observation difficult. Moreover, the quasibrittle damage spreading from a straight notch (i.e., the increase of the microcracked PZ size and/or the increase in microcracks density within the PZ) is expected to lead to an anomalous scaling rather than a Family-Vicsek one, as reported for wood [20] and mortar [16]. The fact that the value of the roughness exponent is observed close to 0.8 in very different materials with various damage processes as, e.g., plastic deformation, crack blunting, ductile cavity growth or microcracking is in agreement with the suggestion [30] of a universal correlated gradient percolation process (i.e., independent of the precise nature of the damage). To test this scenario experimentally will represent interesting challenges for future investigations.

\section{ACKNOWLEDGMENTS}

We thank G. Mourot for providing the experimental results on mortar rupture. This work has received financial support from the Région Aquitaine.
[1] B. B. Mandelbrot, D. E. Passoja, and A. J. Paullay, Nature (London) 308, 721 (1984).

[2] E. Bouchaud, J. Phys.: Condens. Matter 9, 4319 (1997).

[3] E. Bouchaud, Surf. Rev. Lett. 10, 797 (2003).

[4] E. Bouchaud, G. Lapasset, and J. Planés, Europhys. Lett. 13, 73 (1990).

[5] R. H. Dauskardt, F. Haubensk, and R. O. Ritchie, Acta Metall. Mater. 38, 143 (1990).

[6] A. Imre, T. Pajkossy, and L. Nyikos, Acta Metall. Mater. 40, 1819 (1992).

[7] S. Morel, T. Lubet, J.-L. Pouchou, and J.-M. Olive, Phys. Rev. Lett. 93, 065504 (2004).

[8] J. J. Mecholsky, D. E. Passoja, and K. S. Feinberg-Ringel, J. Am. Ceram. Soc. 72, 60 (1989).

[9] K. J. Måløy, A. Hansen, E. L. Hinrichsen, and S. Roux, Phys. Rev. Lett. 68, 213 (1992).
[10] L. Ponson, D. Bonamy, and E. Bouchaud, Phys. Rev. Lett. 96, 035506 (2006).

[11] D. Bonamy, L. Ponson, S. Prades, E. Bouchaud, and C. Guillot, Phys. Rev. Lett. 97, 135504 (2006).

[12] L. Ponson, D. Bonamy, and L. Barbier, Phys. Rev. B 74, 184205 (2006).

[13] L. Ponson, Ann. Phys. 32, 1 (2007).

[14] J. Schmittbuhl, F. Schmitt, and C. Scholz, J. Geophys. Res. 100, 5953 (1995).

[15] J. M. López and J. Schmittbuhl, Phys. Rev. E 57, 6405 (1998).

[16] G. Mourot, S. Morel, E. Bouchaud, and G. Valentin, Phys. Rev. E 71, 016136 (2005).

[17] G. Mourot, S. Morel, E. Bouchaud, and G. Valentin, Int. J. Fract. 140, 39 (2006)

[18] J. Weiss, Eng. Fract. Mech. 68, 1975 (2001).

[19] T. Engøy, K. J. Måløy, A. Hansen, and S. Roux, Phys. Rev. 
Lett. 73, 834 (1994).

[20] S. Morel, J. Schmittbuhl, J. M. López, and G. Valentin, Phys. Rev. E 58, 6999 (1998).

[21] S. Morel, G. Mourot, and J. Schmittbuhl, Int. J. Fract. 121, 23 (2003).

[22] I. L. Menezes-Sobrinho, M. S. Couto, and I. R. B. Ribeiro, Phys. Rev. E 71, 066121 (2005).

[23] A. S. Balankin, O. Susarrey, and J. Marquez González, Phys. Rev. Lett. 90, 096101 (2003).

[24] N. Mallick, P.-P. Cortet, S. Santucci, S. G. Roux, and L. Vanel, Phys. Rev. Lett. 98, 255502 (2007).

[25] E. Bouchbinder, I. Procaccia, S. Santucci, and L. Vanel, Phys. Rev. Lett. 96, 055509 (2006).

[26] J. M. Boffa, C. Allain, and J.-P. Hulin, Eur. Phys. J.: Appl. Phys. 2, 281 (1998).

[27] L. Ponson, H. Auradou, M. Pessel, V. Lazarus, and J.-P. Hulin, Phys. Rev. E 76, 036108 (2007).
[28] E. Bouchbinder, I. Procaccia, and S. Sela, Phys. Rev. Lett. 95, 255503 (2005).

[29] L. Ponson, H. Auradou, P. Vié, and J.-P. Hulin, Phys. Rev. Lett. 97, 125501 (2006).

[30] A. Hansen and J. Schmittbuhl, Phys. Rev. Lett. 90, 045504 (2003).

[31] S. Morel, J. Schmittbuhl, E. Bouchaud, and G. Valentin, Phys. Rev. Lett. 85, 1678 (2000).

[32] J. M. López and M. A. Rodríguez, Phys. Rev. E 54, R2189 (1996).

[33] J. M. López, M. A. Rodríguez, and R. Cuerno, Phys. Rev. E 56, 3993 (1997).

[34] Z. P. Bažant and Z. Li, J. Eng. Mech. 122, 458 (1996).

[35] S. Ramanathan, D. Ertas, and D. S. Fisher, Phys. Rev. Lett. 79, 873 (1997).

[36] F. Family and T. Vicsek, Dynamics of Fractal Surfaces (World Scientific, Singapore, 1991). 